\title{
Impact on survival of nuclear atypia in epithelioid malignant mesothelioma
}

The authors declare no finacial disclosure

\begin{abstract}
Introduction: Malignant pleural mesothelioma is a rare tumour with a bad prognosis. The only consensual prognostic factors are represented by the stage and the histologic type. Concerning the histologic type, epithelioid mesothelioma is known to have better prognosis in comparison with the sarcomatoid and biphasic types. Epithelioid mesotheliomas have been reported to be a heterogeneous prognostic group. Our aim was to assess the impact on the survival of different characteristics of epithelioid mesothelioma, including nuclear atypia, mitotic count, MIB-1 index, inflammatory host response, stromal desmoplasia, necrosis, vascular emboli and invasion depth. Material and methods: We performed a study of survival of 30 malignant pleural mesotheliomas according to the different parameters studied.

Results: The study included 26 women and 4 men. The mean age of the patients was 61 years. The microscopic exam concluded to an epithelioid mesothelioma in 17 cases, sarcomatoid mesothelioma in 4 cases and biphasic mesothelioma in 9 cases. The 17 cases of epithelioid mesothelioma developed severe nuclear atypia in 6 cases and mild nuclear atypia in 11 cases. The mitotic count and the MIB-1 score were respectively inferior to 5 mitoses/50 HPF and inferior to $10 \%$ in 11 cases and were superior to 5 mitoses/50 HPF and superior to 10\% in 6 cases. No vascular emboli were recorded. Tumour necrosis was reported in 1 case. The inflammatory host response was severe in 4 cases and mild in 13 cases. The tumoral stroma was desmoplastic in 4 cases. The invasion depth was superior to $0.5 \mathrm{~mm}$ in 16 cases. The median overall survival amounted to 180 days. Nuclear atypia was the only feature that had impact on survival in the group of epithelioid mesothelioma.

Conclusion: Our results highlight the correlation of nuclear atypia with survival.
\end{abstract}

Key words: malignant mesothelioma, prognosis, epithelioid mesothelioma

Adv Respir Med. 2019; 87: 90-95

\section{Introduction}

Malignant pleural mesothelioma (MPM) is a rare tumour accounting for $2 \%$ of all pleural tumours with a bad prognosis. Its management has been improved by the emergence of new therapeutic modalities, including immunotherapy. The most relevant prognostic factors are represented by the tumour stage and the histologic type. Many modifications have been made in the field of staging of mesothelioma in order to assess a more accurate prognostic category. In fact, some authors reported that the prognostic impact of the 6th and 8th AJCC editions was insufficient [1]. Concerning the typing of mesothelioma, 2 major groups were identified in the 2015 World Health Organization classification including localised and diffuse mesothelioma [2]. Each group contains epithelioid, sarcomatoid and biphasic mesothelioma. Epithelioid mesotheliomas have better prognosis. Among this group, many authors reported an important prognostic heterogeneity. This fact made the researchers look for other prognostic factors necessary in that group [3]. Many authors reported the nuclear grade and necrosis as prognostic factors [4]. Our aim was to assess the 
impact on the survival of different characteristics of epithelioid mesothelioma, including nuclear atypia, mitotic count, MIB-1 index, inflammatory host response, stromal desmoplasia, necrosis, vascular emboli and invasion depth.

\section{Material and methods}

We conducted a retrospective study of 30 MPM diagnosed over a 20-year period (19952015). We included only patients with complete records, including clinical, radiologic and microscopic features.

1. Standard techniques: All the samples were performed after a certain delay of fixation (8 hours for biopsies and 24 hours for surgical specimen).

2. Immunohistochemical technique: Different techniques were applied manually. The used diagnostic antibodies were as follows: anti-calretinin (clone CAL 6, Leica, diluted at 1:200), anti-Epithelial Membrane Antigen (EMA) (clone GP1.4, Leica, diluted at 1:100), anti-mesothelin (clone 5B2, Biogenex, ready-to-use), anti-Thyroid Transcription Factor 1 (TTF1) (clone SPT24, Leica, diluted at 1:100), anti-ACE (clone 12.140.2, Leica, diluted at 1:200), anti-cytokeratin (clones AE1 and AE3 mixed to a ratio 20:1, diluted at 1:100), anti-vimentin (clone V9, Leica, diluted at 1:800), anti-CD15 (clone Carb-1, Leica, ready-to-use), anti-cytokeratin 5/6 (clone CK5/6.007, Biocare, diluted at 1:100), anti-bcl2 (clone bcl 2/100/D5, Leica, diluted at 1:100), anti-CD99 (clone PCB1, Leica, diluted at 1:100) and anti-CD34 (clone QBEnd10, Dako, diluted at 1:25). The scoring of MIB-1 was performed using an anti-Ki67 antibody (clone MIB-1, Immunotech, Westbrook, ME, USA, diluted at 1:100).

Microscopic diagnosis: All the microscopic diagnoses were reviewed by 2 pathologists (MM, FM). A mean of 12 slides per case were re-examined. The diagnosis was based on the 2015 WHO classification [2]. In the group of epithelioid and biphasic mesotheliomas, we assessed prognostic criteria reported by Krasinskas M. and colleagues [5].

Nuclear atypia (severe/mild): nuclear features were evaluated using high-power field (HPF) at x400 magnification using an Olympus SX41 microscope (Olympus, Tokyo, Japan) with an eyepiece of $22 \mathrm{~mm}$ diameter (Fig. 1A, B). Mitotic count (high/low): it was evaluated after scanning through all tumour slides and counted as an average of mitotic figures per 50 high-power fields. The cut-off of 5 mitoses was chosen. MIB-1 scoring: MIB-1 index was recorded as the percentage of tumour cells with positive nuclear immunostaining. A cut-off of $10 \%$ was used. Desmoplastic stroma: present or absent (Fig. 1C). Vascular emboli: present or absent. Inflammatory host response: It was evaluated as mild to moderate or severe (Fig. 1D, E). Histologic subtype: The following 5 histologic patterns were recorded in 5\% increments: tubulo-papillary, trabecular, micro-papillary, solid and pleomorphic. Only 3 patterns were observed in our study: the tubulo -papillary growth pattern consisting of tubules associated to papillary structures (Fig. 1F), the trabecular pattern composed of thin cords of tumour cells (Fig. 1G) and solid pattern made up of nests of tumour cells (Fig. $1 \mathrm{H}$ ). Necrosis: present or absent. The depth of invasion: It was evaluated as superficial or deep with a cut-off of $0.5 \mathrm{~mm}$.

A review of the literature was performed on PubMed (http://www.ncbi.nih.gov/pubmed), Google and Google Scholar using the following keywords: malignant mesothelioma, pleural mesothelioma, epithelioid mesothelioma, desmoplastic mesothelioma, biphasic mesothelioma, sarcomatoid mesothelioma.

\section{Statistical analysis}

The different mean/median survival and the survival curves were represented using SPSS statistics 23. The comparison of the survival curves was made using the logRank. The mean of survival was estimated as the interval between the date of diagnosis and the date of death or a point date fixed for $15^{\text {th }}$ March 2018.

\section{Results}

\section{Epidemiologic and clinical characteristics}

The mean age of the patients was 61 years, average 22 to 80 years. The sex ratio (M/F) was 6.5. Asbestos exposure was reported in 21 cases. The most frequent symptom was chest pain reported in 25 cases. Physical exam was normal in 9 cases. It revealed a pleural syndrome in most patients (60\%). Imaging findings consisted mainly in diffuse pleural thickening in 17 cases. The tumours were classified as stage I in 12 cases, stage II in 3 cases, stage III in 14 cases and stage IV in 1 case. Needle pleural biopsy was performed in 18 cases. In the other cases, the biopsy was performed through thoracoscopy in 16 cases and thoracotomy in 3 cases. It allowed the diagnosis in respectively 7 


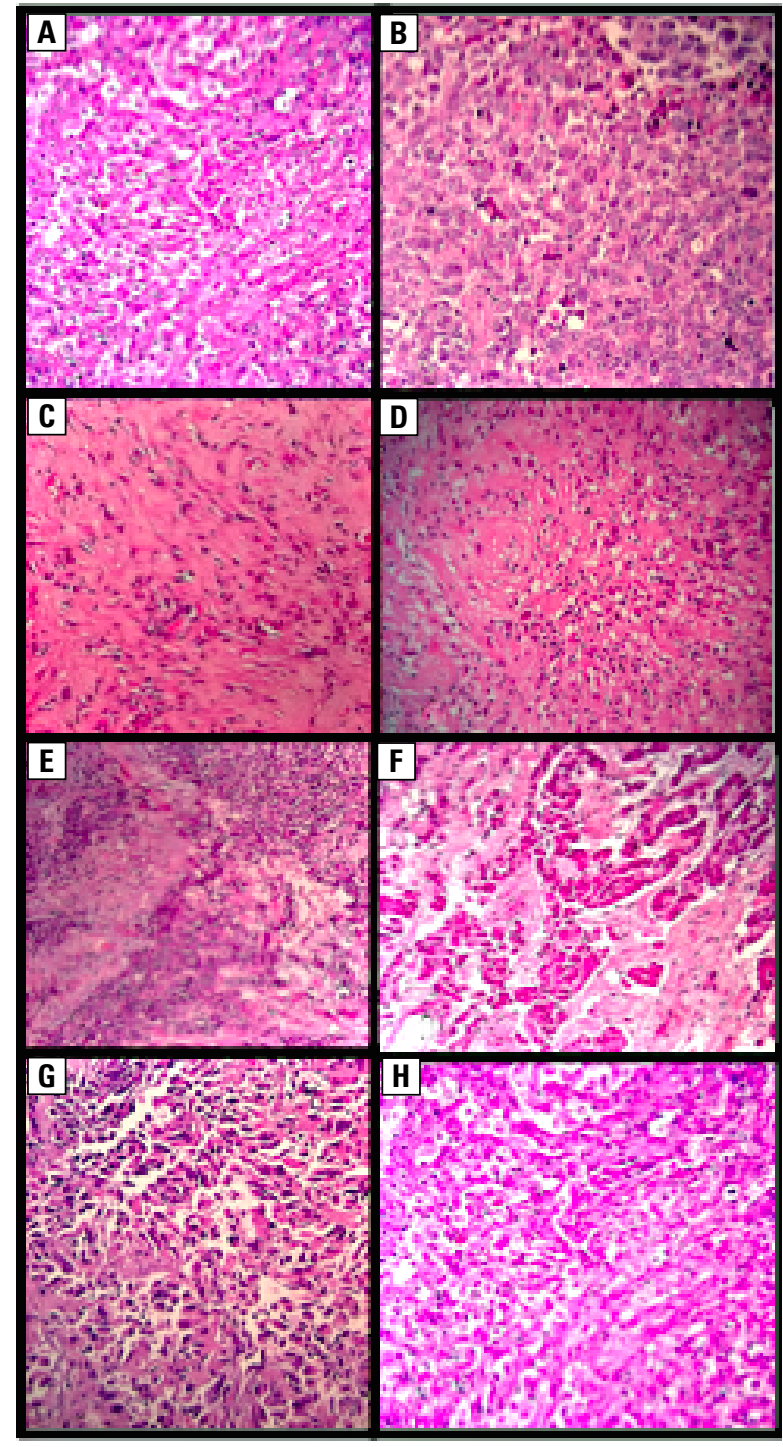

Figure 1. A - mild nuclear atypia in epithelioid malignant mesothelioma (HE $\times 200)$; B - severe nuclear atypia in epithelioid malignant mesothelioma (HE $\times 400)$; C - desmoplastic stroma in an epithelioid malignant mesothelioma (HE $\times 200$ ); D - mild inflammatory host response in an epithelioid malignant mesothelioma (HE $\times 200$ ); $\mathbf{E}$ - severe inflammatory host response in an epithelioid malignant mesothelioma (HE $\times 200$ ); F - papillary epithelioid mesothelioma $(\mathrm{HE} \times 200) ; \mathbf{G}-$ trabecular epithelioid mesothelioma (HE $\times 200)$; H - solid epithelioid mesothelioma (HE $\times 200)$

cases/18, 16 cases/16 and 3 cases/3. A lymph node biopsy was performed through mediastinoscopy in one case and yielded the diagnosis. The diagnosis was made on surgical specimen in 2 patients: one bullectomy and one right upper lobectomy. The Table 1 summarises the epidemiologic and clinical features.

\section{Microscopic features}

The microscopic exam concluded to an epithelioid mesothelioma (EM) in 17 cases, sarcomatoid mesothelioma (SM) in 4 cases and biphasic mesothelioma (BM) in 9 cases. The 17 cases of EM presented severe nuclear atypia in 6 cases and mild nuclear atypia in 11 cases (Fig 1A, B). The mitotic count and the MIB-1 score were respectively inferior to 5 mitoses/50 high power fields and inferior to $10 \%$ in 11 cases and were superior to 5 mitoses/50 high power fields and superior to $10 \%$ in 6 cases. No vasular emboli were recorded. Tumour necrosis was reported in 1 case. The inflammatory host response was severe in 4 cases and mild in 13 cases. The tumoral stroma was desmoplastic in 4 cases. The invasion depth was superior to $0.5 \mathrm{~mm}$ in 16 cases. The Table 2 illustrates the microscopic characteristics of the $17 \mathrm{EM}$.

\section{Follow-up}

Fifteen patients were lost of view after a mean follow-up period of 2.8 months. Thirteen subjects died before or during the treatment after a mean follow-up period of 6.6 months. One patient had a sarcomatoid localised mesothelioma diagnosed on a bullectomy. He developed no complications after a follow-up period of 11 years. One person was treated surgically and suffered from bone metastases after a follow-up period of 4 months.

\section{Survival study}

The median overall survival amounted to 180 days, 95\%CI [63-296] (Fig. 2A)

The median overall survival was estimated at 365 days in stage 1a, 210 days in stage 1b, 60 days in stage 2, 365 days in stage 3a, 180 days in stage $3 b$ and 90 days in stage $4(p=0.423)$. The survival rate was estimated at $66 \%$ at 4 months, in comparison to $0 \%$ at 3 months in stage $2.0 \%$ at 4 months in stage 3 and $0 \%$ at 3 months in stage 4 .

The different characteristics assessed in EM were as follows.

Nuclear atypia: The median survival was estimated at 210 days, CI95\% [0-431] in mild cases and 365 days, CI95\%[36-383] in severe cases. A significant statistical difference was reported between both groups $(p=0.045)$ (Fig. 2B)

Mitotic count: The median survival of patients with high grade was 210 days, CI95\% [0-427] and 365 days in the low-grade group, CI95\% [536-383]. There was no statistical difference between both curves $(p=0.248)$.

MIB-1 index: The median survival was estimated respectively at 210 days in high- grade group IC95\% [0-427] and 365 days IC95\% [536-383] in low-grade group. There was no statistical diffe- 
Table 1. Patients' characteristics

\begin{tabular}{|c|c|}
\hline Age & Mean: 61 years $[22-80$ years] \\
\hline Sex & $26 \mathrm{M} / 4 \mathrm{~W}$ \\
\hline Smoking & 17 patients \\
\hline Asbetos & 21 patients \\
\hline Symptoms & 29 patients symptomatic: chest pain: 25 cases (83.3\%) \\
\hline \multirow[t]{4}{*}{ Imaging } & Chest-x-Ray \\
\hline & $\begin{array}{c}\text { Pleural opacity: } 27 \text { cases }(90 \%) \\
\text { Pleural thickening: } 2 \text { cases }(6.7 \%) \\
\text { Pneumothorax: } 1 \text { case } \\
\text { Associated to: } \\
\text { Cardiomegaly (1 case), right mediastinal opacity (1 case), reticulo-nodular infiltration } \\
\text { (1 case), costal lysis (1 case), alveolar opacity }(1 \text { case) }\end{array}$ \\
\hline & CT-scan \\
\hline & $\begin{array}{c}\text { Diffuse pleural thickening: } 17 \text { cases }(56.7 \%) \text {, tissular mass: } 5 \text { cases }(16.5 \%) \text {, pleural fluid: } \\
19 \text { cases }(63.4 \%) \text {, mediastinal adenomegaly: } 5 \text { cases }(16.7 \%) \text {, atelectasis: } 5 \text { cases }(16.7 \%) \text {, } \\
\text { pleural nodules: } 4 \text { cas }(13.3 \%) \text {, parenchymal condensation: } 2 \text { cases }(6.7 \%)\end{array}$ \\
\hline Stages & 9 stage IA, 3 stage IB, 3 stage II, 14 stage IIIB, 1 stage IV \\
\hline Means of diagnosis & $\begin{array}{l}\text { Pleural cytology: } 7+/ 18 \text {, Needle pleural biopsy: } 7+/ 18 \text {, pleuroscopy: } 1+/ 1 \text {, thoracoscopy: } \\
\qquad 16+/ 16 \text {, thoracotomy: } 3+/ 3 \text {, mediastinoscopy: } 1+/ 1\end{array}$ \\
\hline Microscopic diagnosis & $17 \mathrm{EM}, 4 \mathrm{SM}$ et $9 \mathrm{BM}$ \\
\hline TT and follow-up & $\begin{array}{c}\text { Surgical resection: } 2 \text { cases. } 11 \text { talc pleurodesis }+ \text { chemo } \pm \text { radiation therapy: } 14 \text { cases } \\
15 \text { lost of view } \\
\text { median survival: } 180 \text { days }\end{array}$ \\
\hline
\end{tabular}

EM: epithelioid mesothelioma; SM: sarcomatoid mesothelioma; BM: biphasic mesothelioma

Table 2. Microscopic characteristics of the epithelioid mesotheliomas

\begin{tabular}{lc}
\hline Microscopic features & Number of cases \\
\hline Nuclear atypia & $\begin{array}{c}\text { Severe: } 6 \text { cases } \\
\text { Mild: } 11 \text { cases }\end{array}$ \\
Mitotic count & $<5$ mitoses/50HPF: 11 cases \\
& $>5$ mitoses $/ 50 \mathrm{HPF}: 6$ cases \\
MIB-1 index & $<10 \%: 11$ cases \\
Vascular emboli & $>10 \%: 6$ cases \\
Inflammatory host response & 0 \\
Desmoplastic stroma & Severe: 4 cases \\
Depth invasion & Mild: 13 cases \\
Necrosis & 4 cases \\
\hline
\end{tabular}

rence between both curves ( $p=0.248$ ) (Fig. 2C). The 4-month survival was estimated at $25 \%$ in the group with low mitotic index in comparison to $16 \%$ in the group with high index.

Histologic subtype: EM was classified into trabecular subtype in 5 cases, tubulo-papillary subtype in 9 cases and solid subtype in 5 cases.
There was no significant difference between all curves ( $p=0.05$ ) (Fig. 2D). In tubulopapillary and trabecular subtypes, we noticed a survival at 2 months of respectively 0 and $40 \%$.

Necrosis: Necrosis was present in 1 case. The survival curves showed no significant statistical difference $(p=0.6)$.

Invasion depth: The invasion thickness was inferior to $0.5 \mathrm{~mm}$ in 2 cases and superior to $0.5 \mathrm{~mm}$ in 17 cases. The survival curves presented no significant statistical difference $(\mathrm{p}=0.05)$.

Inflammatory host response: The mean survival in the group with discrete to mild reaction and the group with severe reaction was estimated respectively at 233 days, 95\%CI [137-329] and 142 days, 95\%CI [78-206] ( $\mathrm{p}=0.9$ ). The survival was estimated at $40 \%$ at 3 months in the group with mild to moderate reaction in comparison to $25 \%$ in the group with severe reaction.

Desmoplastic stroma: The mean survival of patients developing tumours without desmoplastic stroma and with desmoplastic stroma accounted respectively for 271 days, 95\%CI [184-357] and 172 days, 95\%CI [82-262] $(\mathrm{p}=0.686)$. 


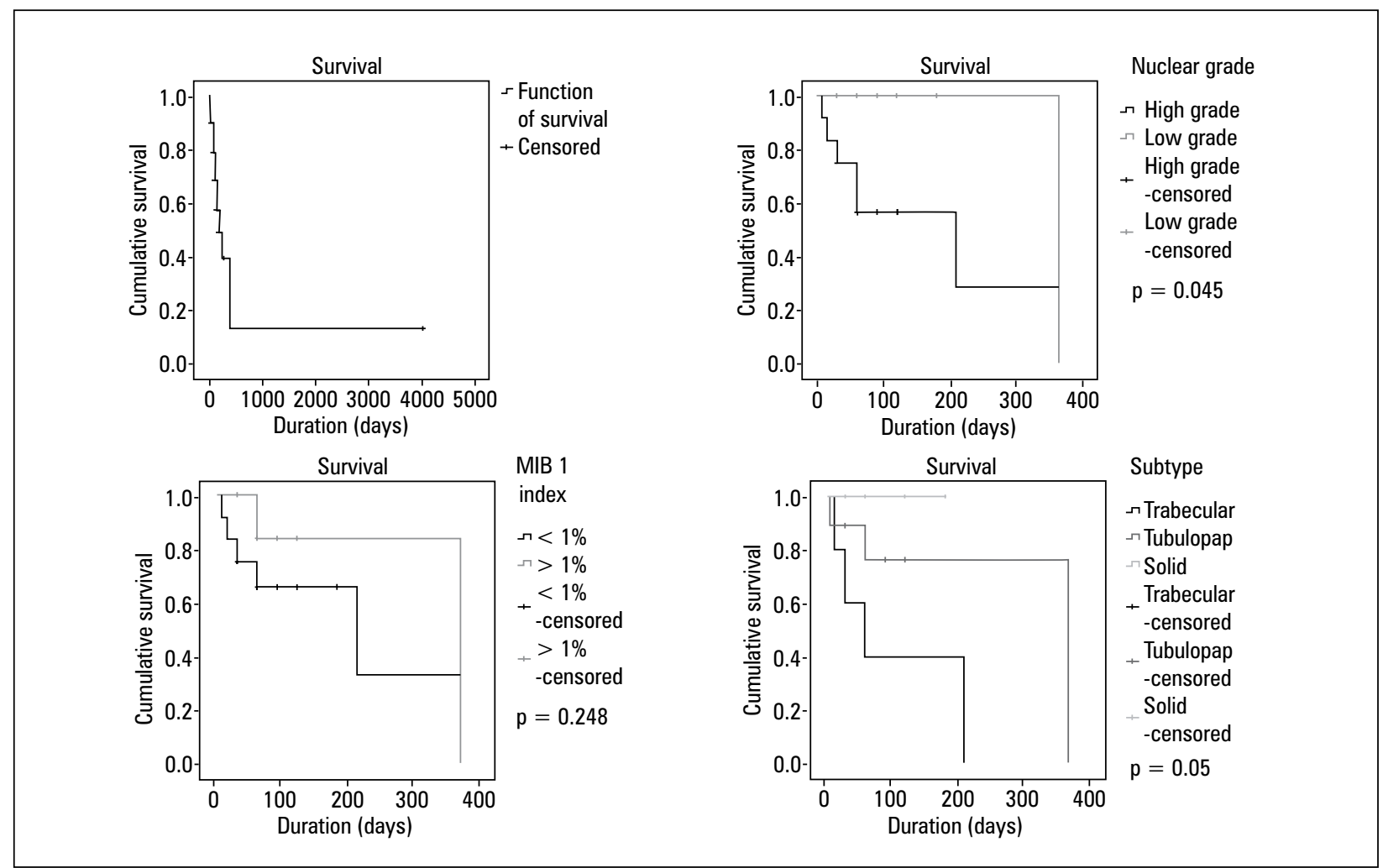

Figure 2. A - survival curve of the 30 patients included in the study; $\mathbf{B}-$ survival curves according to the nuclear atypia $(p=0.045) ; \mathbf{C}-$ survival curves according to the MIB-1 index $(p=0.248) ; \mathbf{D}$ - survival curves according to the histologic subtype $(p=0.05)$

\section{Discussion}

In the study, the authors aimed to assess the prognostic impact of some microsocpic features in epithelioid mesothelioma. Nuclear atypia was the only feature that had prognostic impact. This fact has to be taken with caution because of the important number of lost of view cases in the study. Prognostic factors of MPM have been widely discussed in the literature with varying results and some similarities. Nuclear atypia has been reported as a prognostic factor by some authors and has been integrated into a nuclear grade in association with mitotic atypia by others [4]. Wang et al. [6], published a nomogram to predict prognosis in malignant pleural mesothelioma. The nomogram integrated the age, race, histology type, T stage, M stage, surgical resection and chemotherapy as independent prognostic factors. The stage has been reported as the most reliable prognostic factor. In our study, the prognostic impact of stage wasn't assessed but we noticed that in stage 1 , the survival was estimated at $66 \%$ at 4 months, in comparison to $0 \%$ at 3 months in stage $2,0 \%$ at 4 months in stage 3 and $0 \%$ at 3 months in stage 4 . In the 2015 WHO classification of MPM, Kadota et al. [7] reported the worse prognostic impact of pleomorphic subtype in comparison with the other subtypes, including tubulopapillary, papillary, micropapillary, trabecular, solid, and pleomorphic. In our study, the prognostic impact of the histologic subtype was not assessed because of the crossing survival curves. The only reported subtypes were solid, tubulopapillary and trabecular. All solid subtypes were lost of view but concerning tubulopapillary and trabecular subtypes, we noticed a survival at 2 months of respectively 0 and $40 \%$. Prognostic impact of histologic subtypes has to be assessed in teams trained in the diagnosis of MPM. This is due to variable reproducbility of subtyping reported in the literature. In fact, some authors noted a strong reproducibility with good agreement and others described moderate to substantial agreement [8,9]. In a multi-institutional study, Rosen L. et al. [4], assessed three grading systems consisting in the association of nuclear grade and mitotic count, nuclear grade and necrosis and mitotic count and necrosis [4]. They reported that the nuclear grade predicts survival in epithelioid mesothelioma and that necrosis helps to stratify overall survival. In our study, we tried to establish the prognostic impact of vascular emboli, invasion 
thickness and necrosis but we didn't reach an interpretable result because of the absence of both criteria in almost all cases. Concerning the host reaction, we didn't assess a prognostic impact but we noticed that the survival was estimated at $40 \%$ at 3 months in the group with mild to moderate reaction in comparison to $25 \%$ in the group with severe reaction. Concerning mitotic index, we also noted that the 4-month survival was estimated at $25 \%$ in the group with low index in comparison to $16 \%$ in the group with high index. This tendency was also observed for mitotic index. In fact, the 4-month survival was estimated at $25 \%$ in the group with low mitotic index in comparison to $16 \%$ in the group with high index. Concerning desmoplastic stroma, we noticed that the 4-month survival was estimated at $20 \%$ in the group without desmoplastic stroma in comparison to $25 \%$ in the group with desmoplastic stroma.

\section{Conflict of interest}

The authors declare no conflict of interest.

\section{References:}

1. Abdel-Rahman O. Challenging a dogma; AJCC 8th staging system is not sufficient to predict outcomes of patients with malignant pleural mesothelioma. Lung Cancer. 2017; 113: 128-133, doi: 10.1016/j.lungcan.2017.09.015, indexed in Pubmed: 29110839.
2. Galateau-Salle F, Churg A, Roggli V, et al. World Health Organization Committee for Tumors of the Pleura. The 2015 World Health Organization Classification of Tumors of the Pleura: Advances since the 2004 Classification. J Thorac Oncol. 2016; 11(2): 142-154, doi: 10.1016/j.jtho.2015.11.005, indexed in Pubmed: 26811225.

3. Nowak AK, Chansky K, Rice DC, et al. Staging and Prognostic Factors Committee, Advisory Boards and Participating Institutions. The IASLC Mesothelioma Staging Project: Proposals for Revisions of the T Descriptors in the Forthcoming Eighth Edition of the TNM Classification for Pleural Mesothelioma. J Thorac Oncol. 2016; 11(12): 2089-2099, doi: 10.1016/j. jtho.2016.08.147, indexed in Pubmed: 27687963.

4. Rosen LE, Karrison T, Ananthanarayanan V, et al. Nuclear grade and necrosis predict prognosis in malignant epithelioid pleural mesothelioma: a multi-institutional study. Mod Pathol. 2018; 31(4): 598-606, doi: 10.1038/modpathol.2017.170, indexed in Pubmed: 29327706.

5. Krasinskas AM, Borczuk AC, Hartman DJ, et al. Prognostic significance of morphological growth patterns and mitotic index of epithelioid malignant peritoneal mesothelioma. Histopathology. 2016; 68(5): 729-737, doi: 10.1111/his.12807, indexed in Pubmed: 26272336.

6. S. Wang, K. Ma, Z. Chen, X. Yang, and F. Sun, "A Nomogram to Predict Prognosis in Malignant Pleural Mesothelioma," no.; 180: 2017.

7. A. Manuscript and S. Magnitude, "NIH Public Access, " vol. 31, no 9, pp 1713. 1723; 2013

8. Husain AN, Colby TV, Ordóñez NG, et al. Guidelines for Pathologic Diagnosis of Malignant Mesothelioma 2017 Update of the Consensus Statement From the International Mesothelioma Interest Group. Arch Pathol Lab Med. 2018; 142(1): 89-108, doi: 10.5858/arpa.2017-0124-RA, indexed in Pubmed: 28686500.

9. Brčić L, Jakopović M, Brčić I, et al. Reproducibility of histological subtyping of malignant pleural mesothelioma. Virchows Arch. 2014; 465(6): 679-685, doi: 10.1007/s00428-014-1664-9, indexed in Pubmed: 25300229. 